\title{
On Surjectivity for Nonlinear Maps in Banach Spaces $\left(^{*}\right)\left({ }^{1}\right)$.
}

\author{
Massimo FurI (Firenze) ( ${ }^{2}$ ) and Alfonso Vignoli (Cosenza) $\left({ }^{3}\right)$
}

Sunto. - Sia $E$ uno spazio di Banach e $f: E \rightarrow E$ un operatore continuo, non necessariamente lineare. $\$ i$ danno delle condizioni su $f$ affinchè l'equazione $x-f(x)=y$ sia risolubile per ogni $y \in E$.

\section{Introduction.}

Let $f: E \rightarrow E$ be a continuous (possibly nonlinear) map from a Banach space $E$ into itself. We are concerned with finding conditions on $f$ to get surjectivity for the map $1-f$, where $1: E \rightarrow E$ is the identity. This is equivalent to answering the question of whether the equation $x-f(x)=p$ has a solution for any $p \in E$.

This paper is divided into two parts. The first part aims at maximum generality in order to extend and unify many of the previous known results on the subject. In the second part we select the elass of hemibounded maps (see definition below) that are easier to deal with in computations and seem to be handier in applications.

We shall also show how hemibounded maps are related to bifurcation problems in Banach spaces.

We shall now list some known results regarding surjectivity in the sense mentioned above.

a) Let $f: E \rightarrow E$ be a Banach contraction, then $(1-f)(E)=E$.

A continuous map $f: E \rightarrow E$ is said to be asymptotically linear [5] if there exists a bounded linear operator $L: E \rightarrow E$ such that

$$
\lim _{\|x\| \rightarrow \infty} \frac{\|f(x)-L(x)\|}{\|x\|}=0 .
$$

The unique bounded linear operator satisfying this condition is denoted by $f^{\prime}(\infty)$ and is called the asymptotic derivative of $f$.

(*) Entrata in Redazione il 30 dicembre 1975.

(1) This paper was written while the authors where visiting at the Mathematics Department of the University of Bonn in the "Sonderforschungsbereich 72 " programm.

(2) Università di Firenze - Istituto di Matematica «U. Dini * Viale Morgagni 67-A 50134 Firenze.

$\left({ }^{3}\right)$ Università della Calabria - Dipartimento di Matematica - Casella postale 377 87100 Cosenza. 
The following result is implicity contained in M. A. KRASNOSEL'skIJ's book [5].

b) Let $f: E \rightarrow E$ be asymptotically linear and compact. If $\left\|f^{\prime}(\infty)\right\|<1$, then $(1-f)(E)=E$.

Actually, [5] contains the following stronger result.

o) Let $f: E \rightarrow E$ be asymptotically linear and compact. If the spectral radius $r\left(f^{\prime}(\infty)\right)<1$, then $(1-f)(E)=E$.

A continuous map $f: E \rightarrow E$ is said to be quasibounded [4] if

$$
\limsup _{\|x\| \rightarrow \infty} \frac{\|f(x)\|}{\|x\|}=|f|<+\infty \text {. }
$$

The finite number $|f|$ is called the quasinorm of $f$.

The following result is due to A. Granas [4].

d) Let $f: E \rightarrow E$ be compact and quasibounded. If $|f|<1$, then $(1-f)(E)=E$.

It can be easily seen that $d$ ) contains $b$ ) since if $f$ is asymptotically linear then $|f|=\left\|f^{\prime}(\infty)\right\|$. But $d$ ) neither contains $a$ ) nor $\left.c\right)$. A first step in unifying these results was made in [11]. Namely,

e) Let $f: E \rightarrow E$ be condensing and quasibounded. If $|f|<1$, then $(1-f)(E)=E$.

Clearly, $e$ ) contains $a$ ), $b$ ) and $d$ ) since Banach contractions and compact maps are condensing. Nevertheless, e) does not contain $c$ ). A second step in unifying these results was successfully undertaizen in [9] in such a way that $a$ ) through $e$ ) become particular cases of Theorem 2.1 given in [9].

In the first part of this paper we give a suitable approach that will allow us not only to extend and unify all of the above mentioned results (including those contained in [9]) but also a recent result due to H. Amann [1]. Namely,

f) Let $f: E \rightarrow E$ be an asymptotically linear $\alpha$-contraction. Assume that $f^{\prime}(\infty)$ does not have real eigenvalues greater than or equal to 1 (i.e. $\lambda x \neq f^{\prime}(\infty) x$ for all $x \neq 0$ and $\lambda \geqslant 1)$. Then $(1-f)(E)=E$.

Amann's result cannot be obtained from those contained in [9]. It should be remarked that $b), c$ ) and $f$ ) are given in the framework of ordered Banach spaces and stated in terms of the existence of a fixed point for $f$, rather than in terms of surjectivity for $1-f$. It can be shown that the results contained in this paper can be suitably modified to hold in the context of ordered Banach spaces as well.

Finally, let us spend a few words regarding some notations used in this paper.

The greek letter $\alpha$ will stand for the Kuratowski measure of noncompactness. A continuous map $f: E \rightarrow E$ from a Banach space into itself will be called $\alpha$-Lipschitz with constant $K \geqslant 0$ if $\alpha(f(A)) \leqslant K \alpha(A)$ for any bounded subset $A \subset E$. The map $f$ is an $\alpha$-contraction if $0 \leqslant K<1, \alpha$-nonexpansive if $K=1$ and condensing if $\alpha(f(A))<$ 
Massimo Furi - ALfonso Vignoli: On surjectivity for nonlinear, ete. 207

$\langle\alpha(A)$ for any bounded $A \subset E$ with $\alpha(A)>0$. Let us mention that a compact map (sending bounded sets into relatively compact sets) is $\alpha$-Lipschitz with constant $K=0$. Perhaps the simplest example of an $\alpha$-contractive map is the sum of a compact map with a Banach contraction.

\section{1. - Essentially bounded maps, solvahility of nonlinear equations and surjectivity.}

Our first step will consist in introducing a class of maps which contains the quasibounded maps of Granas [4] and the s-quasibounded maps of [9].

Let $E$ be a (real or complex) Banach space and let $f: E \rightarrow E$ be continuous. Given $r>0$ consider the extended real number

$$
b(r, f)=\sup \{\lambda \geqslant 0: \lambda x=f(x), \text { for some } x \in E \text { such that }\|x\|=r\}
$$

if the set of eigenvectors of $f$ with norm equal to $r$ is not empty and $b(r, f)=0$ otherwise (the convention sup $\emptyset=0$ will be used throughout this paper). Denote by

$$
(f)_{0}=\inf \{b(r, f): r>0\} \text {. }
$$

For any $p \in E$ define

$$
(f)_{p}=(f+p)_{0},
$$

where $f+p$ denotes the map $x \mapsto f(x)+p$.

If the following number

$$
(f)=\sup \left\{(f)_{p}: p \in E\right\}
$$

is finite then the map $f$ will be called essentially bounded and $(f)$ is the essential norm of $f$.

In order to state our first result we need some further notations. Let $f: E \rightarrow E$ be a continuous map. Consider the following number.

$$
d(f)=\liminf _{\|x\| \rightarrow \infty} \frac{\|f(x)\|}{\|x\|} .
$$

Define the positive spectral radius of $f$ in the following way

$$
r^{+}(f)=\sup \{\lambda \geqslant 0: d(\lambda-f)=0\}
$$

where $\lambda-f$ stands for the map $\lambda \cdot 1-f$, (1-the identity on $E)$.

Let $f, g: E \rightarrow E$ be two maps. We say that $f$ and $g$ are asymptotically equivalent $(f \sim g)$ if

$$
\lim _{\|x\| \rightarrow \infty} \frac{\|f(x)-g(x)\|}{\|x\|}=0
$$


Clearly, this is an equivalence relation. We shall denote by $\hat{f}$ the equivalence class containing $f$. Observe that $f$ is asymptotically linear if and only if $\hat{f}$ contains a (unique) bounded linear operator $f^{\prime}(\infty)$.

Now we are able to state the following

Proposimion 1.1. - The positive spectral radius of quasibounded maps has the following properties.

(a) $r^{+}(t) \leqslant|f|$

(b) $f \sim g \Rightarrow r^{+}(f)=r^{+}(g)$. In particular, if $f$ is asymptotically linear then $r^{+}(f)=$ $=r^{+}\left(f^{\prime}(\infty)\right)$.

(c) if $L$ is bounded and linear then

$$
r+(L)=\sup \{\lambda \geqslant 0: \lambda \in \sigma(L)\}
$$

where $\sigma(L)$ is the spectrum of $L$.

Proof. - (a) We have

$$
\frac{\|\lambda x-f(x)\|}{\|x\|} \geqslant|\lambda|-\frac{\|f(x)\|}{\|x\|}
$$

Therefore, if $\lambda \geqslant 0$

$$
d(\lambda-f) \geq \lambda-|f|
$$

Thus $a(\lambda-f)=0$ implies $\lambda \leqslant|f|$. Hence $r^{+}(f) \leqslant|f|$.

(b) It is not difficult to show (see [2]) that $f \sim g$ implies $d(f)=d(g)$. Furthermore, if $f \sim g$ then $\lambda-f \sim \lambda-g$ for any $\lambda \in \boldsymbol{R}$. Therefore, in this case, $d(\lambda-f)=d(\lambda-g)$. Hence $r^{+}(f)=r^{+}(g)$.

(c) We have to show that the two numbers

$$
\begin{aligned}
& a=\sup \{\lambda \in \boldsymbol{R}: d(\lambda-L)=0\} \\
& b=\sup \{\lambda \in \boldsymbol{R}: \lambda \in \sigma(L)\}
\end{aligned}
$$

are equal. It was pointed out in [3] that $d(\lambda-L)=0$ if and only if $\lambda \in \sigma_{\pi}(L)$, the approximate point spectrum of $L$. Hence there exists a sequence $\left\{x_{n}\right\}$ in $E$ such that $\left\|x_{n}\right\|=1, \forall n \in N$ and $\lambda x_{n}-f\left(x_{n}\right) \rightarrow 0$ as $n \rightarrow+\infty$. Therefore, since $\sigma_{n}(L)$ is closed, $a \in \sigma_{n}(L) \subset \sigma(L)$. This implies that $a \leqslant b$. From the definition of $b$ it follows that $b \in \partial \sigma(L)$-the boundary of $\sigma(L)$. On the other hand it is well known that $\partial \sigma(L) \subset \sigma_{n}(L)$, hence $b \leqslant a$, and we are done. Q.E.D.

In the following proposition we list some properties of essentially bounded maps. 
Proposition 1.2:

(a) $(\lambda f)=\lambda(f)$ for any $\lambda \geqslant 0$.

(b) If $f$ is quasibounded then $f$ is essentially bounded and the following estimates of $(f)$ hold

$$
(f) \leqslant r^{+}(f) \leqslant|f|
$$

(c) If $L$ is a bounded linear operator with $\alpha$-Lipschitz constant $K<r^{+}(L)$ then $(L)_{0}=(L)=r^{+}(L)$.

Proof. $-(a)$ We may assume $\lambda>0$. We have

$$
(\lambda f)_{p}=(\lambda f+p)_{0}=\left(\lambda\left(f+\lambda^{-1} p\right)\right)_{0}=\lambda\left(f+\lambda^{-1} p\right)_{0}=\lambda(f)_{\lambda^{-1} p} .
$$

Hence

$$
(\lambda f)=\sup \left\{(\lambda f)_{p}: p \in E\right\}=\sup \left\{\lambda(f)_{\lambda^{-1} p}: p \in E\right\}=\lambda(f)
$$

(b) On the basis of Proposition 1.1- (a) we have only to prove the inequality $(f) \leqslant r^{+}(f)$.

Observe that $r^{+}(f+p)=r^{+}(f)$ for any $p \in E$. Therefore it is enough to prove that $(f)_{0} \leqslant r^{+}(f)$.

If $(f)_{0}=0$ the assertion is trivial. Assume $(f)_{0}>0$. From the definition of $(f)_{0}$ it follows that given $0<a<(f)_{0}$ and $n \in N$ we can find $x_{n} \in E, \lambda_{n} \geqslant a$ such that $\left\|x_{n}\right\|=n$ and $\lambda_{n} x_{n}=f\left(x_{n}\right)$. Hence,

$$
\lambda_{n}=\frac{\left\|f_{n}\left(x_{n}\right)\right\|}{\left\|x_{n}\right\|}
$$

Since $f$ is quasibounded this implies that $\left\{\lambda_{n}\right\}$ is bounded. Therefore, we may assume that $\left\{\lambda_{n}\right\}$ converges to some $\lambda \geqslant a$.

Now,

$$
\frac{\left\|\lambda x_{n}-f\left(x_{n}\right)\right\|}{\left\|x_{n}\right\|} \leqslant\left|\lambda_{n}-\lambda\right|+\frac{\left\|\lambda_{n} x_{n}-f\left(x_{n}\right)\right\|}{\left\|x_{n}\right\|}=\left|\lambda_{n}-\lambda\right|
$$

Thus,

$$
\frac{\left\|\lambda x_{n}-f\left(x_{n}\right)\right\|}{\left\|x_{n}\right\|} \rightarrow 0 \quad \text { as } \quad n \rightarrow+\infty
$$

This means $d(\lambda-f)=0$, i.e. $r^{+}(f) \geqslant \lambda \geqslant a$. Since $a$ can be chosen arbitrarily close to $(f)_{0}$ we get $(f)_{0} \leqslant r^{+}(f)$.

(c) Since $(L)_{0} \leqslant(L) \leqslant r^{+}(L)$ it is enough to show that $(L)_{0} \geqslant r^{+}(L)$. We may assume $r^{+}(L)>0$. As in the proof of Proposition 1.1-(e) it can be shown that $r^{+}(L) \in$ 
$\in \sigma_{\pi}(L)$ - the approximate point spectrum of $L$. So there exists a sequence $\left\{x_{n}\right\}$ in $E$ with $\left\|x_{n}\right\|=1$, such that $r^{+}(L) x_{n}-L\left(x_{n}\right)$ goes to zero as $n \rightarrow+\infty$. Consider the set $A=\left\{x_{n}: n \in \mathbf{N}\right\}$. Clearly,

$$
r^{+}(L) \alpha(A)=\alpha(L(A)) \leqslant K \alpha(A) .
$$

Since $r^{+}(L)>K$ we get $\alpha(A)=0$, i.e. $A$ is relatively compact. We may therefore assume that $\left\{x_{n}\right\}$ is convergent to some $x$ such that $\|x\|=1$. By the continuity of $L$ we get $r^{+}(L) x=L(x)$, i.e. $r^{+}(L)$ is an eigenvalue for $L$. On the other hand the linearity of $L$ implies that $(L)_{0}$ is the largest positive eigenvalue for $L$. Therefore $(L)_{0} \geqslant r^{+}(L)$. Q.E.D.

The following is the main result of this section.

THEOREM 1.1. - Let $f: E \rightarrow E$ be condensing.

(a) If $(f)_{0}<1$, then $f$ has a fixed point.

(b) If $(f)_{p}<1$, then the equation $x-f(x)=p$ has at least a solution $x \in E$.

(c) If $(f)<1$, then $(1-f)(E)=E$.

Proof. - Clearly, $(a) \Rightarrow(b) \Rightarrow(c)$. Therefore we have only to prove $(a)$. The inequality $(f)_{0}<1$ implies the existence of $r>0$ such that $b(r, f)<1$. Let $R: E \rightarrow$ $\rightarrow B(0, r)$ be the radial retraction onto the ball $B(0, r)$ centered at the origin and radius $r$, i.e.

$$
R(x)= \begin{cases}x & \text { if }\|x\| \leqslant r \\ \frac{r x}{\|x\|} & \text { if }\|x\| \geqslant r\end{cases}
$$

Since $R$ is $\alpha$-nonexpansive (see R. D. NUssbaUm [6]) the map $R$ of is condensing and sends $B(0, r)$ into itself and hence it has a fixed point $x \in B(0, r)$. If $\|f(x)\| \leqslant r$ then $x$ is a fixed point for $f$. If $\|f(x)\|>r$ then $x=(r \cdot f(x)) /\|f(x)\|$. Thus

$$
f(x)=\frac{\|f(x)\|}{r} \cdot x \quad \text { with } \quad \frac{\|f(x)\|}{r}>1,
$$

contradicting the fact that $b(r, f)<1$. Q.E.D.

Theorem 1.1 contains as particular cases all the results $a$ ) through $e$ ) and represents a direct extension of Theorem 2.1 of [9] since the essential norm is smaller than or equal to the s-quasinorm introduced in [9]. It also contains Amann's result $f$ ). We shall show this in the sequel.

As an immediate consequence of Proposition 1.1, Proposition 1.2 and Theorem 1.1 we have the following.

CoRollaky 1.1. - Let $f: E \rightarrow E$ be an asymptotically linear condensing map. If $r^{+}\left(f^{\prime}(\infty)\right)<1$ then $(1-f)(E)=E$. 
REMARK 1.1. - If $r\left(f^{\prime}(\infty)\right)$ stands for the spectral radius of $f^{\prime}(\infty)$ then we obviously have $r^{+}\left(f^{\prime}(\infty)\right) \leqslant r\left(f^{\prime}(\infty)\right) \leqslant\left\|f^{\prime}(\infty)\right\|$. The last inequality shows that the results $b$ ) and c) mentioned in the introduction of this paper are direct consequences of Corollary 1.1. We shall show that Corollary 1.1 contains $f$ ) as well.

We need the following.

LEMMA 1.1. - Let $L: E \rightarrow E$ be a linear $\alpha$-contraction. Assume that $L$ satisfies the following condition

$$
\lambda x \neq L(x) \quad \text { for all } x \neq 0 \text { and } \lambda \geqslant 1 \text {. }
$$

Then $r^{+}(L)<1$.

Proof. - It was proved by R. D. Nussbaum [8] that if $L$ is $\alpha$-Lipsehitz with constant $K$ then $\lambda \in \sigma(L)$ and $|\lambda|>K$ imply that $\lambda$ is an eigenvalue for $L$. On the other hand condition $(a)$ means that $L$ has not positive eigenvalues greater than or equal to 1 . Hence $r^{+}(L)<1$. Q.E.D.

We are now in a position of proving Amann's result [1].

COROLLaRY 1.2. - Let $f: E \rightarrow E$ be an asymptotically linear $\alpha$-contraction. Let the asymptotic derivative $f^{\prime}(\infty)$ of $f$ satisfy condition (a) of Lemma 1.1. Then $1-f$ is onto.

Proof. - It is known (see [1] or [9]) that if $f$ is $\alpha$-contractive then so is $f^{\prime}(\infty)$. By Lemma 1.1 we have $r^{+}\left(f^{\prime}(\infty)\right)<1$. Now apply Corollary 1.1. Q.E.D.

The next is an example showing that neither Granas $e$ ) nor Amann's result $f$ ) apply while Theorem 1.1 is applicable.

Example 1.1. - Let $f: \boldsymbol{C} \rightarrow \boldsymbol{C}$ be defined as follows $f(z)=z \cdot \exp (i / \sqrt{1+|z|})$ for any $z \in C$. In this case $(f)=0$. Indeed, we have to solve the equation $\lambda z-f(z)=p$, $p \in \boldsymbol{C}$. In other words if $z \neq 0$ we have

$$
\lambda=\exp \left(\frac{i}{\sqrt{1+|z|}}\right)+\frac{p}{2} .
$$

By setting $z=r \exp (i \theta), p=\varrho \exp (i \varphi)$ we get

$$
\lambda=\exp \left(\frac{i}{\sqrt{1+r}}\right)+\frac{\varrho}{r} \exp (-i(\theta-\varphi)) .
$$

Therefore

$$
\lambda=\cos \frac{1}{\sqrt{1+r}}+i \sin \frac{1}{\sqrt{1+r}}+\frac{\varrho}{r} \cos (\theta-\varphi)-\frac{i \varrho}{r} \sin (\theta-\varphi) .
$$


Obviously, since $\lambda$ is real we must have

$$
\sin \frac{1}{\sqrt{1+r}}-\frac{\varrho}{r} \sin (\theta-\varphi)=0
$$

This equation is solvable iff

$$
\left|\sin \frac{1}{\sqrt{1+r}}\right| \leqslant \frac{\varrho}{r},
$$

which is elearly impossible for $r$ large enough. Therefore $(f)=0$. Now observe that the function $f$ is asymptotically linear and its asymptotic derivative $f^{\prime}(\infty)$ is the identity on $\boldsymbol{C}$. Clearly, $f$ does not satisfy neither Granas condition since $|f|=1$ nor Amann's condition since 1 is an eigenvalue of $f^{\prime}(\infty)$. On the other hand $(f)=0$ and thus Theorem 1.1 applies.

\section{2. - Hemibounded maps, properties and its relation to bifurcation on Banach spaces.}

In this part we introduce the class of hemibounded maps which is included in the class of essentially bounded maps. However, hemibounded maps are easier to deal with and have nice properties that relate them to bifurcation problems in Banach spaces.

Let $f: E \rightarrow E$ be a continuous map from a Banach space $E$ into itself and let $b(r, f)$ be defined as in the first part of this paper.

Set

$$
\langle f\rangle_{0}=\limsup _{r \rightarrow+\infty} b(r, f)
$$

Note that (as for $\left.(f)_{0}\right)$ in the case when $f$ is linear $\langle f\rangle_{0}$ is the supremum of all positive eigenvalues of $f$.

In analogy to what we have done in the first part of the paper we put for any $p \in E$

$$
\langle f\rangle_{p}=\langle f+p\rangle_{0}
$$

The mapping $f$ is said to be hemibounded if the following number is finite

$$
\langle f\rangle=\sup \left\{\langle f\rangle_{p}: p \in E\right\}
$$

From the definition of $\langle f\rangle$ it follows immediately that $(f) \leqslant\langle f\rangle$.

Other properties of hemibounded maps are gathered in the following 
Proposition 2.1:

(a) $\langle\lambda f\rangle=\lambda\langle f\rangle$ for any $\lambda>0$.

(b) If $f$ is quasibounded then $f$ is hemibounded and the following estimates of $\langle f\rangle$ hold

$$
\langle f\rangle \leqslant r^{+}(f) \leqslant|f|
$$

(c) If $L$ is bounded linear and $\alpha$-Lipschitz with constant $K<r^{+}(L)$ then $(L)=$ $=\langle f\rangle=r^{+}(L)$.

The proof of Proposition 2.1 is analogous to the one of Proposition 1.2.

The following is an example showing that the strict inequality $(f)\langle\langle f\rangle$ may indeed occur.

ExampLe 2.1. - Let $f: \boldsymbol{R} \rightarrow \boldsymbol{R}$ be defined by $f(x)=x \operatorname{sen}^{2} x$. Note that in the case of real functions

$$
b(r, f)=\max \left\{0, \frac{f(r)}{r},-\frac{f(-r)}{r}\right\}
$$

In our case

$$
b(r, f+p)=\operatorname{sen}^{2} r+\frac{|p|}{r}, \quad \forall p \in \boldsymbol{R} .
$$

Hence,

$$
\begin{aligned}
& \langle f\rangle_{\mathfrak{p}}=\limsup _{r \rightarrow+\infty}\left(\operatorname{sen}^{2} r+\frac{|p|}{r}\right)=1, \\
& (f)_{p}=\inf _{r>0}\left(\operatorname{sen}^{2} r+\frac{|p|}{r}\right)=0 .
\end{aligned}
$$

Thus, $(f)=0$ and $\langle f\rangle=1$.

REMark 2.1. - Theorem 1.1 holds for hemibounded maps by simply substituting everywhere in the corresponding statements essential boundedness with hemiboundedness.

In order to prove the next result we need the following lemma which represents an extension of the well-known Birkoff-Kellog theorem to the context of $\alpha$-Lipschitz maps.

LemMa 2.1. (Birkoff-Kellog theorem for $\alpha$-Lipsehitz maps). - Let $f: S \rightarrow E$ be an $\alpha$-Lipschitz map from the unit sphere $S=\{x \in E:\|x\|=1\}$ into a Banach space $E$. Assume $\operatorname{dim} E=+\infty$ and

$$
\|f(x)\| \geqslant \varrho>K, \quad \text { for all } x \in S,
$$


where $K \geqslant 0$ is the $\alpha$-Lipschitz constant of $f$. Then $f$ has at least a positive eigenvalue (greater than or equal to $\varrho$ ).

Proof. - Denote by $\bar{U}(\varrho)=\{x \in E ;\|x\| \geqslant \varrho\}$.

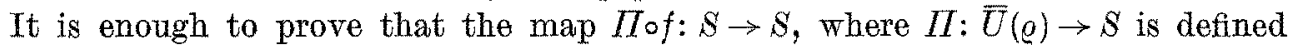
by $\Pi(x)=x /\|x\|$, has a fixed point. To this end we have to show that $\Pi$ of is $\alpha$-contractive and apply Nussbaum's theorem [7]. Observe that $\Pi$ can be regarded as the composition $\Pi=s \circ r$, where $r$ is the radial retraction of $\bar{U}(\varrho)$ onto $S(\varrho)=\{x \in E:\|x\|=$ $=\varrho\}$ and $s: S(\varrho) \rightarrow S$ is defined by $s(x)=x / \varrho$. Now, the map $\Pi l \circ f=s$ or of is $\alpha$-contractive with constant $K / \varrho<1$ since $f$ is $\alpha$-Lipschitz with constant $K$, the map $r$ is $\alpha$-nonexpansive (see R. D. NUsSBaUm [6]) and the map s satisfies $\alpha(s(A))=(1 / \varrho) \alpha(A)$ for all $A \subset S(\varrho)$. Q.E.D.

The following proposition relates $\langle f\rangle_{0}$ with

$$
d(f)=\liminf _{\|x\| \rightarrow+\infty} \frac{\|f(x)\|}{\|x\|}
$$

introduced in the first part of this paper.

Proposition 2.2. - Let $f: E \rightarrow E$ be $\alpha$-Lipschitz with constant $K$. Assume $\operatorname{dim} E=$ $=+\infty$ and $d(f)>K$. Then $d(f) \leqslant\langle f\rangle_{0}$.

Proof. - Let $c$ be such that $K<c<d(f)$. It is enough to show that there exists $r_{0}>0$ such that for all $r>r_{0}$ there is at least an eigenvalue $\lambda$ of $f$ on $S(r)=$ $=\{x \in E:\|x\|=r\}$ such that $\lambda \geqslant c$. In fact, if this is the case we have $\langle f\rangle \geqslant c$ and $c$ can be chosen arbitrarily close to $d(f)$. Now, from the definition of $d(f)$ we can find $r_{0}>0$ such that $\|x\| \geqslant r_{0}$ implies $\|f(x)\| \geqslant e\|x\|$. At this point for any $r>r_{0}$ apply Lemma 2.1 to the map $f_{r}: S \rightarrow E$ defined by $f_{r}(x)=(1 / r) f(r x)$. Q.E.D.

REMARK 2.2. - The following simple example shows that in the assumptions of Proposition 2.2 we cannot remove neither $d(f)>K$ nor $\operatorname{dim} E=+\infty$. In fact, let $f=-1$ ( 1 is the identity on $E)$. Clearly $d(f)=1$ and $\langle f\rangle=0$. On the other hand the $\alpha$-Lipschitz constant of -1 is 0 if $\operatorname{dim} E<+\infty$ and 1 if $\operatorname{dim} E=+\infty$.

The following proposition shows the existing relation between hemibounded maps and asymptotic bifureation points. We recall that $\lambda \in \boldsymbol{R}$ is called asymptotic bifurcation point (see [5]) of a map $f: E \rightarrow E$ from a Banach space $E$ into itself if there exists a sequence $\left\{\left(\lambda_{n}, x_{n}\right)\right\}$ in $\boldsymbol{R} \times E$ such that $\lambda_{n} \rightarrow \lambda,\left\|x_{n}\right\| \rightarrow+\infty$ and $\lambda_{n} x_{n}-f\left(x_{n}\right)=0$. The set of all asymptotic bifurcation points of $f$ will be denoted with the symbol $B(f)$.

Proposition 2.3. - Let $f: E \rightarrow E$ be hemibounded. Then $\langle f\rangle_{0}=B^{+}(f)=\max \{\lambda \geqslant$ $\geqslant 0: \lambda \in B(f)\}$.

Proof. - To prove $\langle f\rangle_{0} \leqslant B^{+}(f)$ we may assume $\langle f\rangle_{0}>0$. In this case we can find two sequences $\left\{x_{n}\right\}$ in $E$ and $\left\{\lambda_{n}\right\}$ in $\boldsymbol{R}$ such that $\left\|x_{n}\right\| \rightarrow+\infty, \lambda_{n} \rightarrow\langle f\rangle_{0}$ and $\lambda_{n} x_{n}=f\left(x_{n}\right)$. This means that $\left\langle f_{0}\right\rangle$ is an asymptotic bifurcation point of $f$. It remains 
Massimo FurI - Aufonso VIgnoli: On surjectivity for nonlinear, ete. 215

to show that there are no asymptotic bifurcation points greater than $\langle f\rangle_{0}$. Let $\lambda>0$ be an asymptotic bifurcation point of $f$, then there exists two sequences $\left\{x_{n}\right\} \subset E$, $\left\{\lambda_{n}\right\} \subset \boldsymbol{R}$ such that $\lambda_{n} \rightarrow \lambda,\left\|x_{n}\right\| \rightarrow+\infty$ and $\lambda_{n} x_{n}=f\left(x_{n}\right)$. This implies that

$$
b\left(\left\|x_{n}\right\|, f\right) \geqslant \lambda_{n} .
$$

Thus, $\lim _{r \rightarrow+\infty} \sup _{\infty} b(r, f) \geqslant \lambda$, i.e. $\langle f\rangle_{0} \geqslant \lambda$. Q.E.D.

Notice that if $\left.\langle f\rangle_{0}\right\rangle_{0}$ then $B(f) \neq \emptyset$ and $\langle f\rangle_{0}$ is the largest positive asymptotic bifurcation point for $t$.

From Proposition 2.3 and Theorem $2.1-(a)$ it follows that if $f: E \rightarrow E$ is hemibounded, condensing and fixed point free then $f$ has at least an asymptotic bifurcation point $\lambda \geqslant 1$. On the other hand if a map $f: E \rightarrow E$ is not hemibounded then there exist two sequences $\left\{\lambda_{n}\right\}$ in $\boldsymbol{R}$ and $\left\{x_{n}\right\}$ in $E$ such that $\lambda_{n} \rightarrow+\infty,\left\|x_{n}\right\| \rightarrow+\infty$ and $\lambda_{n} x_{n}=f\left(x_{n}\right)$. When this occurs we say that $+\infty$ is an (improper) asymptotic bifurcation point for $f$. We have therefore the following.

COROLLARY 2.1. - Let $f: E \rightarrow E$ be condensing and fixed point free. Then $f$ has a (possibly improper) asymptotic bifurcation point $\lambda \geqslant 1$.

This result in the case when $f$ is compact is an equivalent formulation of Schaefer's theorem [10].

Using Proposition 2.3 we can now prove the estimate $\langle f\rangle \leqslant r^{+}(f)$ without quasiboundedness assumptions on $f$ (see Proposition 2.1-(b)). Namely, we have the following.

Corollary 2.2. - Let $f: E \rightarrow E$ be hemibounded. Then $\langle f\rangle \leqslant r^{+}(f)$.

Proor. It is enough to show that $\langle f\rangle_{0} \leqslant r^{+}(f)$. In fact, if this is true, then $\langle f\rangle_{p}=$ $=\langle f+p\rangle_{0} \leqslant r^{+}(f+p)=r^{+}(f)$. Now, by Proposition 2.3 it sufficies to show $B^{+}(f) \leqslant$ $\leqslant r^{+}(f)$. Without loss of generality we may assume $B^{+}(f)=\lambda>0$. We have $\lambda_{n} x_{n}=$ $=f\left(x_{n}\right)$, where $\lambda_{n} \rightarrow \lambda$ and $\left\|x_{n}\right\| \rightarrow+\infty$. Therefore

$$
\frac{\left\|x_{n}-f\left(x_{n}\right)\right\|}{\left\|x_{n}\right\|}=\left|\lambda-\lambda_{n}\right| \rightarrow 0
$$

as $n \rightarrow+\infty$. Thus $d(\lambda-f)=0$, hence from the definition of $r^{+}(f)$ we get $\lambda \leqslant$ $\leqslant r^{+}(f)$. Q.E.D.

\section{REFERENCES}

[1] H. AMANN, Fixed points of asymptotically linear maps in ordered Banach spaces, Journ. Funct. Anal., 14, no. 2 (1973), pp. 162-171.

[2] M. Forr - A. Vrgnour, A nonlinear spectral approach to surjectivity in Banach spaces, Journ. Funct. Anal. 20, No. 4 (1975), pp. 304-318. 
[3] K. GeORG - M. MARTELL, On spectral theory for nonlinear operators, to appear in Journ. Funct. Anal.

[4] A. Granas, The theory of compact vector fields and some of its applications to topology of functional spaces, Rozprawy Matematyczne, Warszawa, no. 30 (1962), 93 pages.

[5] M. A. Krasnosel'skiJ, Positive solutions of operator equations, Noordhoff, Groningen (1964).

[6] R. D. Nussbaum, The fixed point index for local condensing maps, Ann. Mat. Pura Appl., 89 (1971), pp. 217-258.

[7] R. D. Nussbaum, Some fixed points, Bull. Amer. Math. Soc., 77 (1971), pp. 360-365.

[8] R. D. Nussbaum, The radius of the essential spectrum, Duke Math. Journ., 37 (1970), pp. $473 \cdot 470$.

[9] J. PEJSAChOWICZ - A. VxGNOLI, On differeniability and surjectivity of $\alpha$-Lipschitz maps, Ann. Mat. Pura Appl., 101 (1974), pp. 49-63.

[10] H. H. SChaEFen, Über die Methode der a priori Schranlen, Math. Ann., 129 (1955), pp. $415 \cdot 416$.

[11] A. VIGNOLI, On quasibounded mappings and nonlinear functional equations, Accad. Naz. Lincei, 50, no. 2 (1971), pp. 114-117. 